\title{
Prevention of Proliferative Diabetic Retinopathy and Cataract in SDT Rats with Aminoguanidine, an Anti-Advanced Glycation End Product Agent
}

\author{
Fumihiko Toyoda ${ }^{1}$, Akihiro Kakehashi ${ }^{*}, 1$, Ayumi Ota ${ }^{1}$, Nozomi Kinoshita ${ }^{1}$, Chiho Kambara ${ }^{1}$, \\ Hiroko Yamagami ${ }^{1}$, Hiroyuki Tamemoto ${ }^{2}$, Hiroto Ueba $^{2}$, Yoh Dobashi ${ }^{2}$, San-e Ishikawa ${ }^{2}$, \\ Masanobu Kawakami ${ }^{2}$ and Yasunori Kanazawa ${ }^{2}$
}

\author{
${ }^{I}$ Department of Ophthalmology, Jichi Medical University, Saitama Medical Center, Saitama, Japan \\ ${ }^{2}$ Department of Integrated Medicine I, Jichi Medical University, Saitama Medical Center, Saitama, Japan \\ ${ }^{3}$ Japanese Diabetes Foundation, Tokyo, Japan
}

\begin{abstract}
Advanced glycation end products (AGEs) play important roles in the development of ocular complications in diabetes mellitus. Spontaneously Diabetic Torii (SDT) rats have marked hyperglycemia and severe ocular complications. We evaluated the effect of anti-AGE agents, aminoguanidine and pyridoxamine, and an antioxidant, probucol, on the development of diabetic retinopathy (DR) and cataract in SDT rats. Experiment 1 included five SDT rats treated with aminoguanidine, four SDT rats treated with probucol, and four untreated control SDT rats. After age 55 weeks, we evaluated DR by fluorescein angiomicroscopy and pathological study and cataract by biomicroscopy. Experiment 2 included six SDT rats treated with pyridoxamine, and six SDT rats and 10 non-diabetic normal Sprague-Dawley (SD) rats not treated with pyridoxamine. Retinopathy and cataract were evaluated as in experiment 1 . Urinary pentosidine and Maillard reaction product X (MRX) levels were measured for 40 weeks in each group. Experiment 1: Mature cataracts and DR developed in all untreated SDT rats; aminoguanidine prevented cataracts and DR ( $<<0.05, v s$ untreated SDT rats). Probucol had no effect. Experiment 2: Mature cataracts developed in all untreated SDT rats $(\mathrm{p}<0.001$ vs normal SD rats $[0 / 10])$ and DR developed in $67 \%(\mathrm{p}<0.01, v s$ normal SD rats $[0 / 10,0 \%])$. Pyridoxamine did not prevent cataracts $(6 / 6$, $100 \%)$ or DR $(4 / 6,37 \%)$ (nonsignificant $v s$ untreated SDT rats) in SDT rats. Urinary pentosidine levels were higher in untreated $(0.12 \pm 0.07 \mu \mathrm{g} / \mathrm{mg} \cdot \mathrm{Cre})$ and pyridoxamine-treated $(0.12 \pm 0.05 \mu \mathrm{g} / \mathrm{mg} \cdot \mathrm{Cre})$ SDT rats than normal SD rats $(0.069 \pm 0.019 \mu \mathrm{g} / \mathrm{mg} \cdot \mathrm{Cre})$, but not significantly so. Urinary MRX levels were significantly $(\mathrm{p}<0.01)$ lower in normal SD rats $(17.5 \pm 9.6 \mu \mathrm{g} / \mathrm{mg} \cdot \mathrm{Cre})$ compared with untreated SDT rats $(163.0 \pm 107.0 \mu \mathrm{g} / \mathrm{mg} \cdot \mathrm{Cre})$; pyridoxamine had no effect $(149.0 \pm 66.5 \mu \mathrm{g} / \mathrm{mg} \cdot \mathrm{Cre})$ (nonsignificant $v s$ untreated SDT rats). Aminoguanidine but not pyridoxamine and probucol prevents DR and cataracts in SDT rats.
\end{abstract}

Keywords: Diabetes, diabetic retinopathy, cataract, advanced glycation end products (AGEs), SDT rat.

\section{INTRODUCTION}

Diabetic ocular complications, such as diabetic retinopathy (DR), cataract, and neovascular glaucoma, impair quality of life due to severe visual loss. DR, one of the most serious complications of diabetes mellitus, frequently leads to blindness [1-3]. Despite laser treatment and vitreous surgery, which significantly decrease the likelihood of blindness in patients with diabetes, DR still remains the most frequent cause of postnatal blindness. Proliferative diabetic retinopathy (PDR), the end stage of $\mathrm{DR}$, is characterized by new vessel formation accompanied by fibrous tissue proliferation leading to vitreous hemorrhage, subsequent tractional retinal detachment, and visual loss. Recent studies have suggested that several metabolic disorders following long-term poor glycemic

*Address correspondence to this author at the Department of Ophthalmology, Jichi Medical University, Saitama Medical Center, 1-847 Amanuma-cho, Omiya-ku, Saitama, 330-8503, Japan; Tel: +81-48-647-2111; Fax: +81-6485188; E-mail: kakeaki@omiya.jichi.ac.jp control, including increased polyol pathway activity [4, 5], activation of protein kinase $\mathrm{C}$ (PKC) [6-8], increased oxidative stress $[9,10]$, and accumulation of advanced glycation end products (AGEs) [11, 12], may underlie development of DR. If patients with diabetes are treated with intensive glycemic control from the early stage of the disease, the complications can be prevented. However, not all patients can be diagnosed with diabetes in its early stage or receive appropriate treatment. Moreover, many patients with diabetes do not monitor their blood glucose level in the early stage because of the absence of symptoms. In these situations, new drugs to prevent diabetic complications regardless of the glycemic control have been long desired. However, to test the effectiveness of drugs against PDR, an animal model of PDR is needed. Although numerous diabetic animal models have been described, none has spontaneously developing PDR that closely mimics human DR. We reported a new spontaneously diabetic Torii rat (SDT rat) with severe ocular complications $[13,14]$. In the current study, we tested two anti-AGE agents, aminoguanidine and pyridoxamine, and an antioxidant, 
probucol, in this rat model to evaluate the effects on ocular complications.

\section{Animals}

The SDT rat, a substrain of the Sprague-Dawley (SD) rat, spontaneously develops diabetes mellitus. In 1988, five male rats with polyuria and glucosuria were identified among 305 rats from an outbred colony of the Crj:CD (SD) strain (Charles River Japan, Inc., Kanagawa, Japan) of SD rats. After the 20th generation of sister-brother matings, the diabetic strain was established in 1997. The characteristics of this rat have been described previously [13, 14]. Briefly, male rats develop marked hyperglycemia (about $700 \mathrm{mg} / \mathrm{dl}$ ) and glucosuria after 20 weeks of age. The cumulative incidence of diabetes is almost $100 \%$ by 40 weeks of age. Female rats also develop diabetes; however, it appears much later, i.e., after 45 weeks of age, and the cumulative incidence is only $35 \%$ even after 60 weeks of age. The survival rates of untreated male and female SDT rats up to 65 weeks are 93\% and 97\%, respectively. Mature diabetic cataracts are observed after 40 weeks of age in most male SDT rats. Large retinal folds mimicking a tractional retinal detachment and extensive fluorescein leakage around the optic disc are the most prominent findings of DR in old (mostly after 50 weeks of age) SDT rats. Young SDT rats (under 50 weeks of age) usually do not develop advanced DR.

\section{METHODS}

We performed two experiments to evaluate the effects of aminoguanidine, pyridoxamine, and probucol. The care and handling of all animals were in accordance with the Association for Research in Vision and Ophthalmology Statement for the Use of Animals in Ophthalmic and Vision Research.

\section{Experiment 1}

The SDT rats received regular chow until they developed diabetes, estimated by high blood glucose levels $(>250$ $\mathrm{mg} / \mathrm{dL}$ ). After the rats were confirmed to be diabetic (mean age, 35 weeks), five SDT rats were treated with aminoguanidine $(0.5 \mathrm{~g} / \mathrm{L}$ in drinking water $)$ and four SDT rats were treated with probucol (1\% in pellets). Four SDT rats were left untreated. After 55 weeks of age, fluorescein angiomicroscopy and pathological study were performed, and cataract was evaluated in each group.

\section{Fluorescein Angiomicroscopy}

Fluorescein angiomicroscopy was performed after intracardiac injection of fluorescein-dextran (fluorescein isothiocyanate-dextran, Sigma, St. Louis, MO), using a modification of a previously reported method [13]. Under deep anesthesia induced by intraperitoneal injection of pentobarbital sodium ( $25 \mathrm{mg} / \mathrm{kg}$ body weight), $1 \mathrm{ml}$ of phosphate buffered saline containing $50 \mathrm{mg}$ of fluoresceindextran was injected into the left ventricle of each animal. After 5 minutes, the right eye was enucleated for fluorescein microscopy and the left eye for histopathology. The retina was peeled from the eyecup and the entire retina was flatmounted on a slide glass without fixation. A drop of aqueous mounting medium (Crystal/Mount, Biomeda Corp., Foster City, CA, USA) was applied over the retina and allowed to dry. The flat-mounted retina was examined by fluorescence microscopy (Nikon SMZ1500 with P-FLA fluorescence attachment, Nikon, Tokyo, Japan).

\section{Histopathology}

The eyes were enucleated for histopathology and placed in a fixative (a mixture of $2.5 \%$ paraformaldehyde and $1 \%$ glutaraldehyde in $0.15 \mathrm{M}$ phosphate buffer) to avoid artificial retinal detachment. The fixed eyes were washed in $0.1 \% \mathrm{~mol} / \mathrm{l}$ cacodylate buffer and embedded in paraffin. The paraffin block was sectioned to $4 \mu \mathrm{m}$ and stained with hematoxylin and eosin for conventional histopathologic examination. The immunohistochemical procedures were based on the standard avidin-biotin horseradish peroxidase method using each antibody and developed with AEC Substrate Chromogen (DakoCytomation, Carpinteria, CA, USA). Carboxymethyl lysine (CML) was immunostained with a monoclonal antibody of CML (Trans Genic Inc., Kumamoto, Japan). Bovine serum was used as the primary antibody for negative control of the immunostaining.

\section{Biomicroscopy of Cataract}

The pupils were fully dilated with a topical ophthalmic solution containing tropicamide $5 \%$ and phenylephrine hydrochloride 5\%, and the anterior segment including the lens was observed and photographed in both eyes of all rats.

\section{Experiment 2}

The SDT rats were treated as in experiment 1 except for the drugs studied. After the rats were confirmed to be diabetic, six SDT rats were treated with pyridoxamine $(1 \mathrm{~g} / \mathrm{L}$ in drinking water), and six control SDT rats and 10 nondiabetic normal SD rats were untreated. After 60 weeks of age, fluorescein angiomicroscopy and pathological study were performed and cataract was evaluated as described previously in each group. All rats were housed in metabolic cages for 40 weeks after the onset of diabetes, and a 24-h urine sample was collected. The urine samples were centrifuged at $1,700 \mathrm{xg}$ for $10 \mathrm{~min}$ at $4^{\circ}$ Celsius to obtain the supernatant. The urinary pentosidine level was measured by enzyme-linked immunosorbant assay (ELISA) and the urinary Maillard reaction product X (MRX) level was measured by high-performance liquid chromatography (HPLC). The ELISA and HPLC analyses were performed by Fushimi Pharmaceutical Co Ltd., Marugame, Japan. The pentosidine and MRX levels were corrected by the creatinine level in urine and expressed in micrograms per milligram of creatinine.

The prevalence rates of DR and cataract were evaluated by Fisher's exact test of independence. The Student's t-test and Mann-Whitney's U test were used for comparisons between each group. The values are expressed as mean $\pm \mathrm{SD}$, and $\mathrm{p}<0.05$ was considered significant.

\section{RESULTS}

\section{Experiment 1}

Mature cataracts and DR developed in all animals in the untreated SDT rat group $(4 / 4,100 \%)$, but aminoguanidine prevented cataracts and DR $(0 / 5,0 \%)(\mathrm{p}<0.05$ by Fisher's exact test). Probucol had no effect (4/4, 100\%) (nonsignificant $v s$ untreated SDT rats) (Fig. 1). 


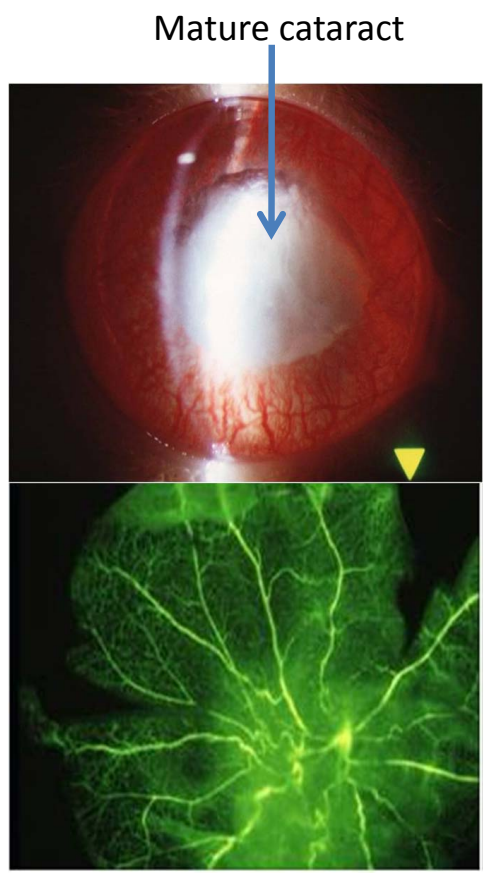

No treatment

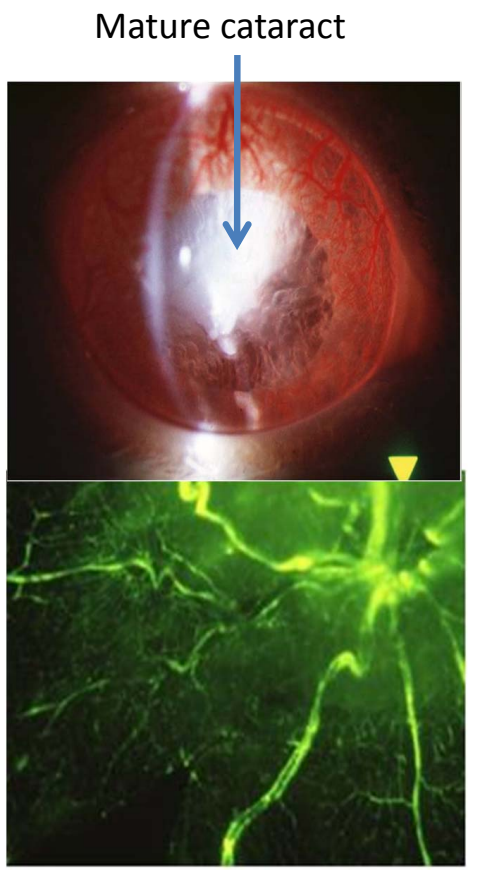

Probucol

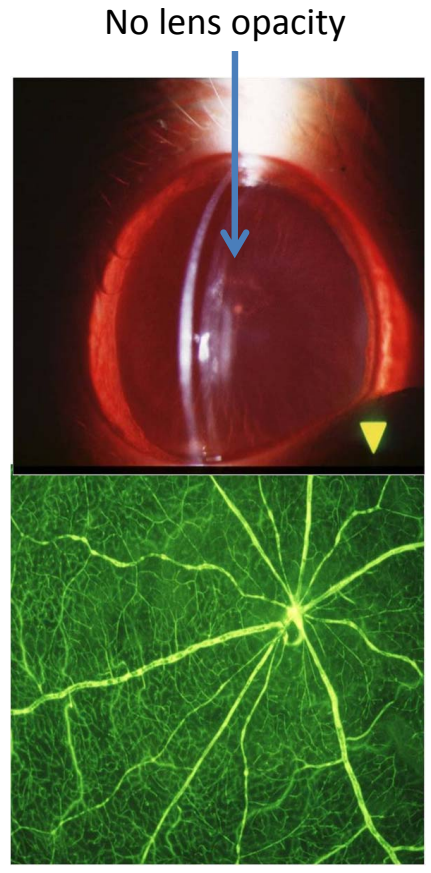

Aminoguanidine

Fig. (1). Effect of aminoguanidine and probucol on diabetic cataract and retinopathy. The top row shows the slit-lamp findings in the lens. The untreated control rat and the probucol-treated SDT rat have mature diabetic cataracts, whereas the aminoguanidine-treated SDT rat has minimal lens opacity. The bottom row shows the fluorescein angiomicroscopy findings. The untreated control rat and the probucoltreated SDT rat have extensive fluorescein leakage and narrowing of the capillaries around the optic disc. The aminoguanidine-treated SDT rat has no abnormalities.

Immunohistochemical study showed less CML staining in the retina and posterior subcapsular space in aminoguanidine-treated eyes compared with probucol-treated eyes (Fig. 2).

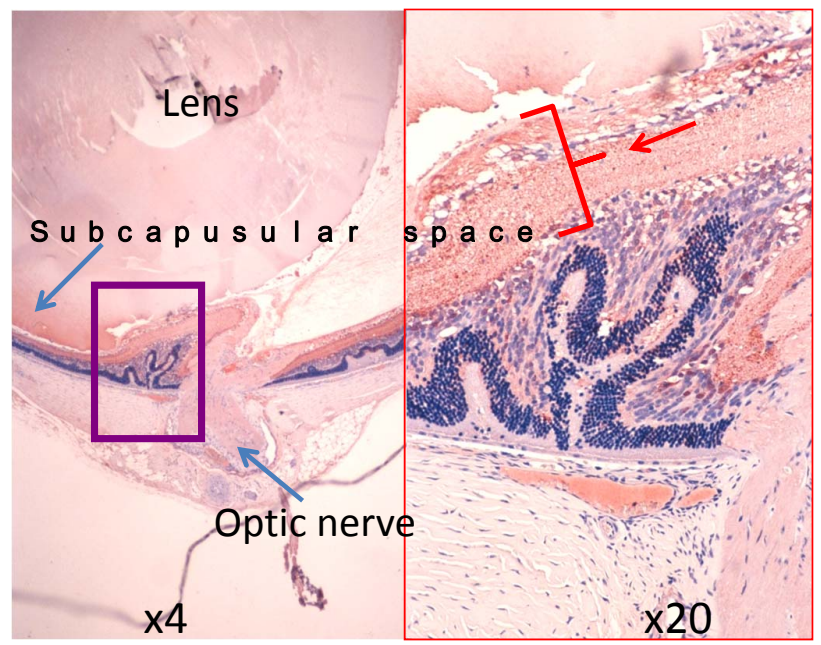

Probucol

\section{Experiment 2}

As in experiment 1, mature cataracts developed in all $(6 / 6,100 \%)$ untreated SDT rats $(\mathrm{p}<0.001$ vs normal SD rats $[0 / 10,0 \%]$ by Fisher's exact test) and DR developed in two thirds $(4 / 6,67 \%)(\mathrm{p}<0.01, v s$ normal SD rats $[0 / 10,0 \%]$ by

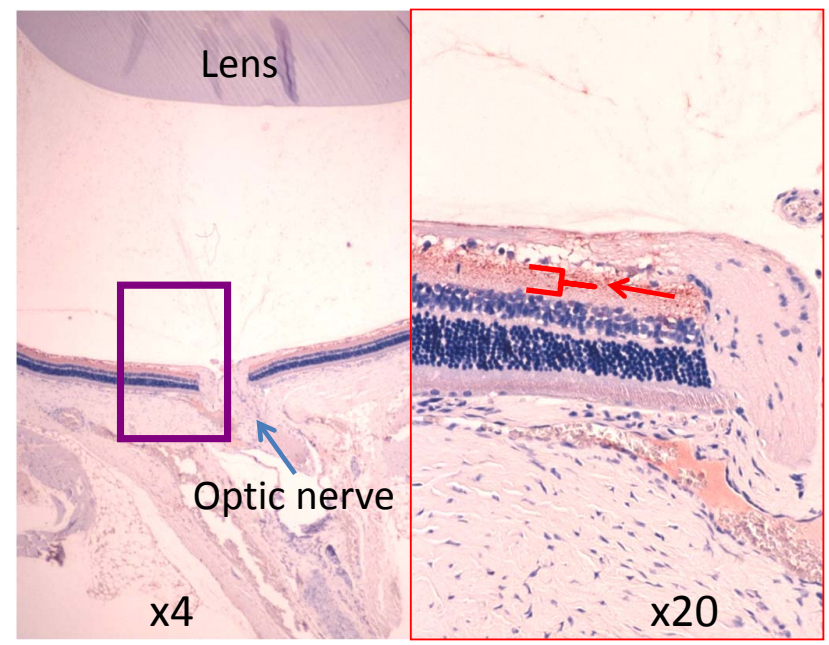

\section{Aminoguanidine}

Fig. (2). Immunohistochemistry for CML in aminoguanidine- and probucol-treated SDT rats. Left, the photographs show extensive staining for CML in the thickened retina and the subcapsular space of the lens in probucol-treated eyes. The magnified photograph shows extensive staining for CML in the inner retina (red arrow). Right, the photographs show less staining for CML in aminoguanidine-treated eyes. The magnified photograph shows no staining for CML in the lens and less staining for CML in the inner retina (red arrow). 
Fisher's exact test). Pyridoxamine did not prevent cataracts in SDT rats $(6 / 6,100 \%)$ or DR $(4 / 6,37 \%)$ (nonsignificant $v s$ untreated SDT rats) (Fig. 3).

Immunohistochemical study showed less staining of CML in the retina and posterior subcapsular space in normal SD rat eyes compared with SDT rat eyes treated with pyridoxamine and those not treated with pyridoxamine (Fig. 4).

The urinary pentosidine levels were not significantly higher in untreated $(0.12 \pm 0.07 \mu \mathrm{g} / \mathrm{mg} \cdot$ creatinine (Cre)) ( $\mathrm{p}=0.13$ by Mann-Whitney's U-test) and pyridoxaminetreated $(0.12 \pm 0.05 \mu \mathrm{g} / \mathrm{mg} \cdot \mathrm{Cre})(\mathrm{p}=0.06$ by Student's t-test $)$
SDT rats than in normal SD rats $(0.069 \pm 0.019 \mu \mathrm{g} / \mathrm{mg} \cdot \mathrm{Cre})$ (Fig. 5).

Urinary MRX levels were significantly $(p<0.01$ by Mann-Whitney's U-test) lower in the normal SD rats $(17.5 \pm 9.6 \mu \mathrm{g} / \mathrm{mg} \cdot \mathrm{Cre})$ compared with the untreated SDT rats $(163.0 \pm 107.0 \mu \mathrm{g} / \mathrm{mg} \cdot \mathrm{Cre})$. Pyridoxamine had no effect $(149.0 \pm 66.5 \mu \mathrm{g} / \mathrm{mg} \cdot \mathrm{Cre}$ ) (nonsignificant vs untreated SDT rats by Student's t-test) (Fig. 6).

\section{DISCUSSION}

Ocular complications in diabetes, cataract, retinopathy, and neovascular glaucoma are thought to result from increased polyol pathway activity, activation of $\mathrm{PKC}$,

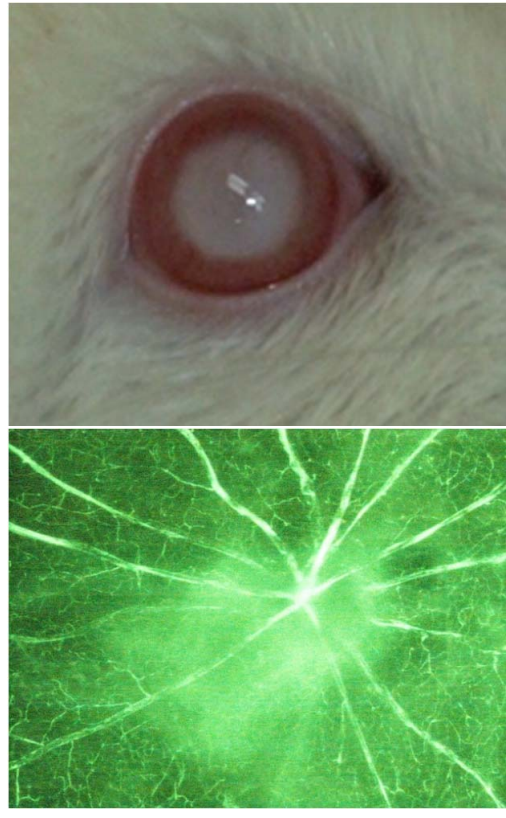

Untreated SDT rat
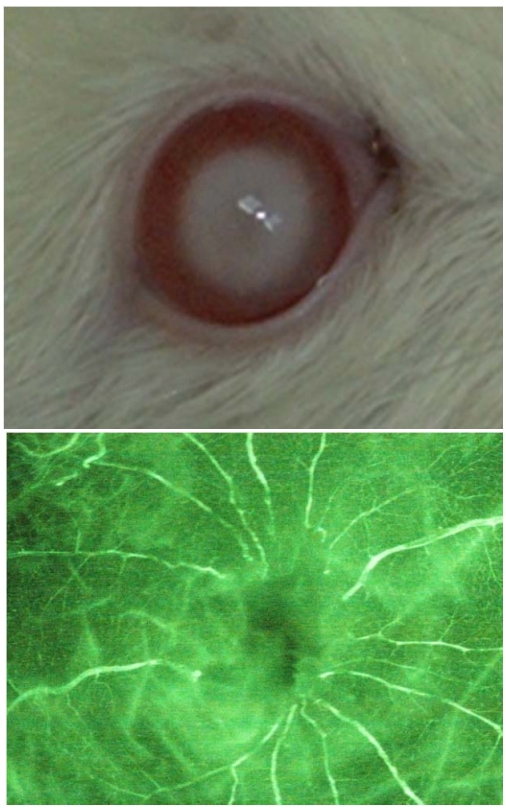

Pyridoxamine-treated SDT rat

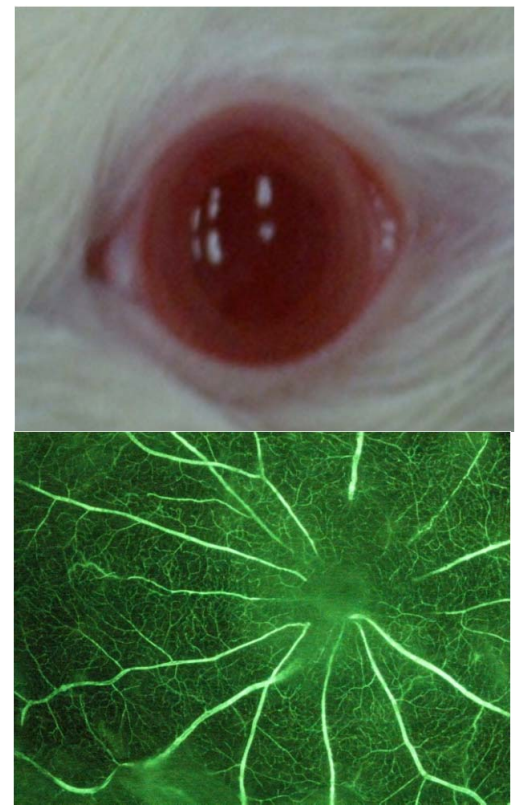

Normal SD rat

Fig. (3). Effect of pyridoxamine on diabetic cataract and retinopathy. The top row shows the biomicroscopic findings in the lens. The untreated control rat and the pyridoxamine-treated SDT rat have mature diabetic cataracts; the normal SD rat has no lens opacity. The bottom row shows the fluorescein angiomicroscopy findings. The untreated control rat and the pyridoxamine-treated SDT rat have extensive fluorescein leakage and narrowing of the capillaries around the optic disc. The normal SD rat has no abnormalities.

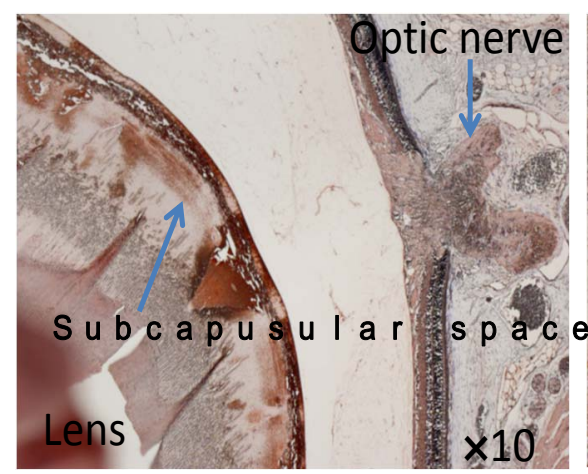

Untreated SDT rat

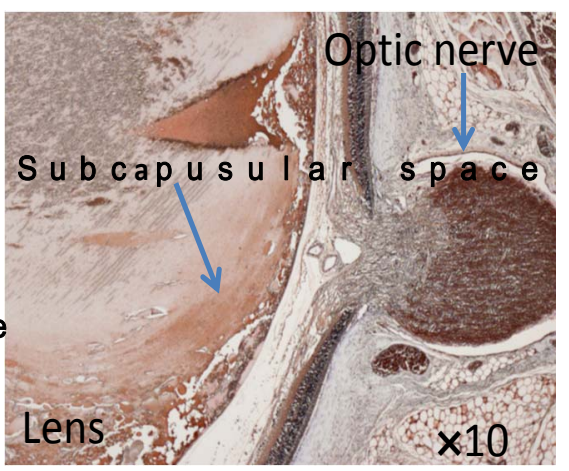

Pyridoxamine-treated SDT rat

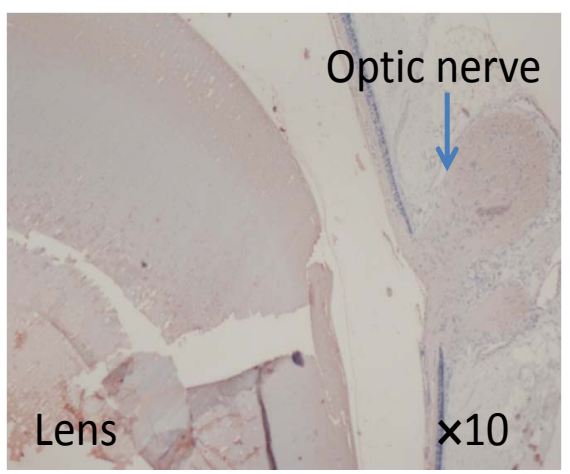

Normal SD rat

Fig. (4). Immunohistochemistry for CML in a pyridoxamine-treated SDT rat. Left and middle, the photographs of the untreated SDT rat and the pyridoxamine-treated SDT rat show extensive staining of CML in the retina and posterior subcapsular space. Right, the photograph of the normal SD rat shows less staining. 


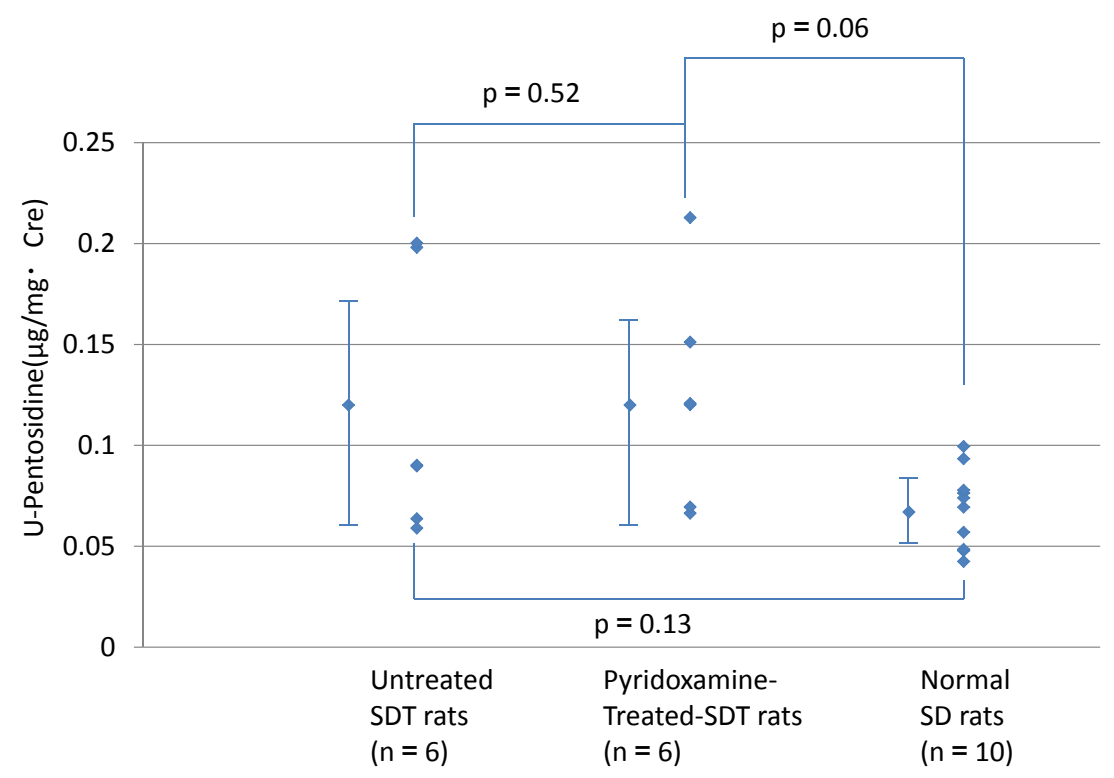

Fig. (5). Urinary pentosidine levels in the pyridoxamine-treated rats. There is no significant difference among the normal SD rats, untreated SDT rats, and pyridoxamine-treated SDT rats.

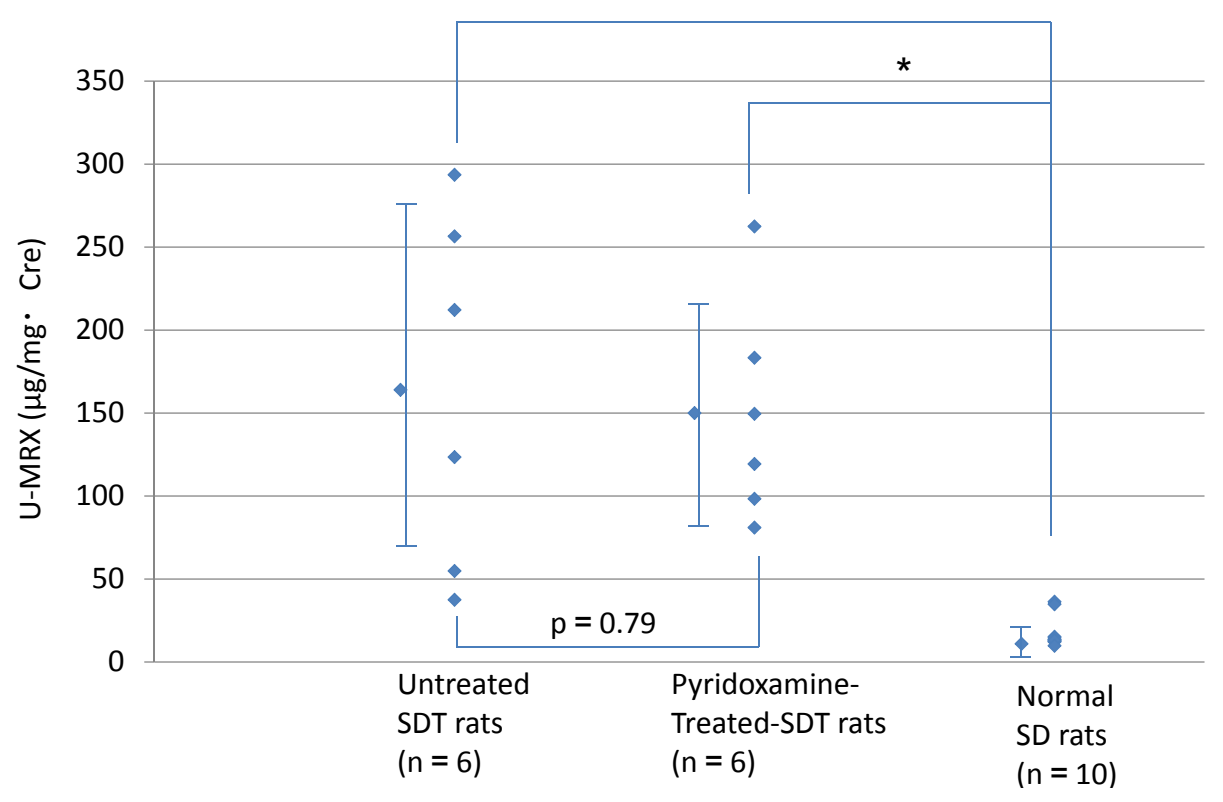

Fig. (6). Urinary MRX levels in the pyridoxamine-treated rats. The urinary MRX levels are lower in normal SD rats than in untreated SDT rats and pyridoxamine-treated SDT rats. There is no significant difference in the MRX levels between the untreated SDT rats and the pyridoxamine-treated SDT rats. * $\mathrm{p}<0.01$ Mann-Whitney U test.

increased oxidative stress, and accumulation of AGEs resulting from prolonged hyperglycemia. In advanced DR, retinal ischemia followed by neovascularization of the retina and/or of iris (neovascular glaucoma) could result in blindness. Among these mechanisms, AGE accumulation is one of the most important. While many compounds are AGEs, CML is a major AGE that affects the ocular complications in diabetes [15-17]. Hammes et al. reported that accumulation of CML is recognized even in early-phase $\mathrm{DR}$ and that increased CML accumulation is associated with progression of DR [18]. However, pentosidine is more important than CML in the development of diabetic nephropathy $[16,19,20]$. So pentosidine is thought to be a marker of nephropathy in patients with diabetes. MRX, a novel AGEs isolated from the hydrolysate of glycated proteins [21], is a good biomarker for detecting oxidative stress produced during the Maillard reaction. In our experiment, pyridoxamine did not decrease pentosidine and MRX in SDT rats.

Several clinical studies have reported that the pentosidine level is related more closely to nephropathy than retinopathy $[16,22]$. Those correlations strongly suggested that CML is the most important AGE-induced neovascularization in the eye. Pyrraline and pentosidine may be other causes of ocular 
complications. Our other publication on immunohistochemical study of SDT rats by Toyoda et al. (Toyoda et al. Accumulation of AGEs and VEGF in Eyes of SDT Rats, TODJ) in the same issue also showed that CML plays an important role in the development of PDR in SDT rats, while pyrraline and pentosidine may not.

Aminoguanidine is a hydrazine compound that prevents formation of AGE by competitive inhibition. Because the early (Amadori-type) glycosylation products have a stronger affinity for aminoguanidine than for lysine $\varepsilon$-amino groups of proteins, they bind to aminoguanidine, inducing formation of unreactive substitutes that cannot crosslink with other similar proteins or trap plasma proteins with which they may be in contact [23]. Moreover, aminoguanidine prevents oxidative modification of low-density lipoprotein [24]. However, pyridoxamine, a member of the $\mathrm{B}_{6}$ vitamer family, is a potent scavenger of reactive carbonyls that inhibits the late stages of glycation reactions that lead to AGE formation [25]. Stitt et al. reported that pyridoxamine inhibits development of DR in streptozotocin (STZ)-induced diabetic rats [26]. In contrast to SDT rats, STZ-induced diabetic rats have only some microaneurysms and thickening of the basement membrane of the retinal capillaries without proliferative changes. However, SDT rats have extensive vascular hyperpermeability and tractional retinal detachment with thickening around the optic disc. Hyperglycemia is more extensive in SDT rats than STZ-induced diabetic rats [14]. Pyridoxamine may regulate DR but may not be effective in serious cases like SDT rats.

In conclusion, aminoguanidine has a strong inhibitory effect on diabetic ocular complications in SDT rats, but probucol and pyridoxamine do not have the same effect. CML seems to be an important AGE in the development of diabetic ocular complications.

\section{REFERENCES}

[1] Hyman L. Epidemiology of eye disease in the elderly. Eye 1987; 1: 330-41.

[2] Krumpaszky HG, Ludtke R, Mickler A, Klauss V, Selbmann HK. Blindness incidence in Germany. A population-based study from Wurttemberg-Hohenzollern. Ophthalmologica 1999; 213: 176-82.

[3] Kocur I, Resnikoff S. Visual impairment and blindness in Europe and their prevention. Br J Ophthalmol 2002; 86: 716-22.

[4] Hotta N, Nakamura J, Sakakibara F, et al. Electroretinogram in sucrose-fed diabetic rats treated with an aldose reductase inhibitor or an anticoagulant. Am J Physiol 1997; 273: E965-71.

[5] Robison WG Jr., Nagata M, Tillis TN, Laver N, Kinoshita JH. Aldose reductase and pericyte-endothelial cell contacts in retina and optic nerve. Invest Ophthalmol Vis Sci 1989 ; 30: 2293-9.

[6] Aiello LP, Bursell SE, Clermont A, et al. Vascular endothelial growth factor-induced retinal permeability is mediated by protein kinase $\mathrm{C}$ in vivo and suppressed by an orally effective betaisoform-selective inhibitor. Diabetes 1997 ; 46: 1473-80.

[7] Lee TS, MacGregor LC, Fluharty SJ, King GL. Differential regulation of protein kinase $\mathrm{C}$ and $(\mathrm{Na}, \mathrm{K})$-adenosine triphosphatase activities by elevated glucose levels in retinal capillary endothelial cells. J Clin Invest 1989; 83: 90-4.
[8] Nakamura J, Kato K, Hamada Y, et al. A protein kinase C-betaselective inhibitor ameliorates neural dysfunction in streptozotocininduced diabetic rats. Diabetes 1999 ; 48: 2090-5.

[9] Altomare E, Grattagliano I, Vendemaile G, Micelli-Ferrari T, Signorile A, Cardia L. Oxidative protein damage in human diabetic eye: evidence of a retinal participation. Eur J Clin Invest 1997; 27: 141-7.

[10] Kuroki M, Voest EE, Amano S, et al. Reactive oxygen intermediates increase vascular endothelial growth factor expression in vitro and in vivo. J Clin Invest 1996 ; 98: 1667-75.

[11] Hammes HP, Wellensiek B, Kloting I, Sickel E, Bretzel RG, Brownlee M. The relationship of glycaemic level to advanced glycation end-product (AGE) accumulation and retinal pathology in the spontaneous diabetic hamster. Diabetologia 1998; 41: 16570 .

[12] Murata T, Nagai R, Ishibashi T, Inomuta H, Ikeda K, Horiuchi S. The relationship between accumulation of advanced glycation end products and expression of vascular endothelial growth factor in human diabetic retinas. Diabetologia 1997 ; 40: 764-9.

[13] Kakehashi A, Saito Y, Mori K, et al. Characteristics of diabetic retinopathy in SDT rats. Diabetes Metab Res Rev 2006 ; 22: 45561.

[14] Shinohara M, Masuyama T, Shoda T, et al. A new spontaneously diabetic non-obese Torii rat strain with severe ocular complications. Int J Exp Diabetes Res 2000; 1: 89-100.

[15] Boehm BO, Schilling S, Rosinger S, et al. Elevated serum levels of $\mathrm{N}$ (epsilon)-carboxymethyl-lysine, an advanced glycation end product, are associated with proliferative diabetic retinopathy and macular oedema. Diabetologia 2004; 47: 1376-9.

[16] Hirata K, Kubo K. Relationship between blood levels of Ncarboxymethyl-lysine and pentosidine and the severity of microangiopathy in type 2 diabetes. Endocr J 2004 ; 51: 537-44.

[17] Hammes HP, Brownlee M, Lin J, Schleicher E, Bretzel RG. Diabetic retinopathy risk correlates with intracellular concentrations of the glycoxidation product Nepsilon(carboxymethyl) lysine independently of glycohaemoglobin concentrations. Diabetologia 1999; 42: 603-7.

[18] Hammes HP, Alt A, Niwa T, et al. Differential accumulation of advanced glycation end products in the course of diabetic retinopathy. Diabetologia $1999 ; 42:$ 728-36.

[19] Sugiyama S, Miyata T, Horie K, et al. Advanced glycation endproducts in diabetic nephropathy. Nephrol Dial Transplant 1996; 11 Suppl 5: 91-4.

[20] Weiss MF, Rodby RA, Justice AC, Hricik DE. Free pentosidine and neopterin as markers of progression rate in diabetic nephropathy. Collaborative Study Group. Kidney Int 1998 ; 54: 193-202.

[21] Oya $\mathrm{T}$, Kumon $\mathrm{H}$, Kobayashi $\mathrm{H}$, et al. A novel biomarker for hyperglycemia, MRX isolated from hydrolysate of glycated proteins. Biochem Biophys Res Commun 1998 ; 246: 267-71.

[22] Capeans Tome C, De Rojas Silva MV, Rodriguez-Garcia J, Rodriguez-Segade S, Sanchez-Salorio M. Levels of pentosidine in the vitreous of eyes with proliferative diabetic retinopathy, proliferative vitreoretinopathy and retinal detachment. Graefes Arch Clin Exp Ophthalmol 2005; 243: 1272-6.

[23] Brownlee M, Vlassara H, Cerami A. Nonenzymatic glycosylation products on collagen covalently trap low-density lipoprotein. Diabetes 1985; 34: 938-41.

[24] Philis-Tsimikas A, Parthasarathy S, Picard S, Palinski W, Witztum JL. Aminoguanidine has both pro-oxidant and antioxidant activity toward LDL. Arterioscler Thromb Vasc Biol 1995; 15: 367-76.

[25] Voziyan PA, Metz TO, Baynes JW, Hudson BG. A post-Amadori inhibitor pyridoxamine also inhibits chemical modification of proteins by scavenging carbonyl intermediates of carbohydrate and lipid degradation. J Biol Chem $2002 ; 277$ : 3397-403.

[26] Stitt A, Gardiner TA, Alderson NL, et al. The AGE inhibitor pyridoxamine inhibits development of retinopathy in experimental diabetes. Diabetes 2002; 51: 2826-32. 